\title{
Clinical Application of Sonographic Elasticity Imaging for Aging of Deep Venous Thrombosis
}

\section{Preliminary Findings}

\author{
Jonathan M. Rubin, MD, PhD, Salavat R. Aglyamov, PhD, \\ Thomas W. Wakefield, MD, Matthew O'Donnell, PhD, \\ Stanislav Y. Emelianov, PhD
}

\begin{abstract}
Objective. Aging of deep venous thrombosis is an important and difficult clinical problem. Because it is known that thrombi harden as they mature, we have preliminarily tested sonographic elasticity imaging, a technique that estimates tissue hardness, to age venous thrombi. Methods. Two adult patients with lower extremity thrombi were studied. One had a clinically chronic thrombus (at least 3 years old), whereas the other patient's thrombus was clinically subacute ( 25 days old). We performed freehand compression sonographic scans using a 5-MHz linear array transducer. Phase-sensitive B-scan frames were processed offline by a two-dimensional complex correlation-based adaptive speckletracking technique. The distribution of internal strains in the wall of the vein, thrombus, and surrounding tissue was analyzed. Clot hardness was normalized to the venous wall. Results. The chronic clot was homogeneous, and the strain in the chronic clot was at least 10 times smaller than that in the vessel wall. The subacute clot was much more heterogeneous, and, on average, the strain magnitude in the clot was 3 to 4 times greater than that in the vessel wall. Conclusions. In this preliminary work, the 2 thrombi appeared very different, and these results suggest that elasticity imaging may be able to age deep venous thrombosis. Key words: compression sonography; deep venous thrombosis; elasticity imaging.
\end{abstract}

Abbreviations

DVT, deep venous thrombosis

Received December 4, 2002, from the Departments of Radiology (J.M.R.), Biomedical Engineering (S.R.A., M.O., S.Y.E.), and Surgery (T.W.W.), University of Michigan, Ann Arbor, Michigan USA; and Institute of Mathematical Problems of Biology, Russian Academy of Sciences, Pushchino, Moscow Region, Russia (S.R.A.). Dr Aglyamov and Dr Emelianov are now with the Biomedical Engineering Department, University of Texas at Austin, Austin, Texas USA. Revision requested January 6, 2003. Revised manuscript accepted for publication February 10, 2003.

This work was supported in part by National Institutes of Health grants HL068658, DK47324, HL47401, and HL63148 and by the Ultrasound Group of Siemens Medical Systems, Inc (Issaquah, WA).

Address correspondence and reprint requests to Jonathan M. Rubin, MD, Department of Radiology, University of Michigan, 1500 E Medical Center Dr, Ann Arbor, MI 48109 USA; e-mail: jrubin@umich.edu.
7 he standard diagnostic test for deep venous thrombosis (DVT) is now venous duplex sonography, a combination of standard gray scale imaging with either spectral or color Doppler and compression sonographic scanning. ${ }^{1}$ The roles of gray scale and Doppler imaging are mainly just to locate the veins. The real diagnostic portion of the test is compression sonography, a technique well described in the literature. ${ }^{2}$ This method has proved highly sensitive and specific for the detection of DVTs. ${ }^{2}$ Once performed, however, compression sonography gives little information regarding the age or maturity of the detected thrombus. ${ }^{1,3,4}$ The estimation of age and maturity of DVTs is important for determining the appropriate therapy. ${ }^{5}$

It is generally accepted that as clots mature, they harden. This is because acute clots are largely composed of platelets, fibrin, and neutrophils, which over time are replaced by collagen and mononuclear cells. ${ }^{6,7}$ This tran- 
sition progressively makes clots harder, and the decrease in platelets and increase in collagen composition of thrombi tend to stabilize the thrombi, reducing the risk of a pulmonary embolism. ${ }^{8}$ Thus, a test that assesses hardness would provide an excellent means of determining the maturity of thrombi. Such a test exists. It is called sonographic elasticity imaging or elastography. ${ }^{9,10}$

Sonographic elasticity imaging is a technique that uses tissue deformation to assess the local hardness of the tissues being deformed. ${ }^{9,10}$ When an operator pushes on tissue with a transducer while imaging, as is done with standard compression sonographic imaging, the speckle in the image moves with the push. Tracking the motion of the speckle permits one to determine the relative hardness of the tissues in the image. In the case of DVT, the push would be similar if not identical to the compression that is presently used to diagnose DVT with standard compression sonography. Elasticity imaging has a long history in basic imaging, and it has shown promise in determining the age of induced thrombi in rat inferior vena cavae. ${ }^{11}$ We report our initial experience using sonographic elasticity imaging in evaluating thrombi in the legs of 2 patients with thrombi of known ages.

\section{Materials and Methods}

Each of the patients had known venous thrombosis based on previously performed compression sonographic scans. Each patient was recruited and gave voluntary informed consent under a research protocol that had been approved by the local Institutional Review Board. The first patient was a 48-year-old man who had DVT in the left popliteal and upper calf veins that was at least 3 years old on the basis of yearly compression sonographic studies. An approximately circular, 5-mm-diameter clot in the popliteal vein located $20 \mathrm{~mm}$ below the skin surface was scanned. The second patient was a 42 year-old man with an elliptical, 6-mm axial and 9.5-mm lateral thrombus in the left saphenous vein located $11 \mathrm{~mm}$ below the skin surface. On the basis of the precise nature of the acute onset of the patient's symptoms of pain and swelling, the clot was thought to be 25 days old at the time of the research scan.

Each patient was scanned in the standard position for a compression sonographic study at the location of the thrombus. Patient 1 was scanned in the prone position, and patient 2 was supine. Scans were performed with a Sonoline Elegra sonographic scanner (Siemens Medical Systems, Inc, Issaquah, WA) capable of capturing frames of digital phase-sensitive sonographic signals. We used a 5-MHz (5.0 HDPL 40) linear array transducer for both compression sonography and elasticity imaging. The thrombus-containing veins were scanned in a typical transverse orientation. We scanned at multiple sites across the clots. The number of sites varied depending on the size of the thrombi; however, we separated the sites by at least the width of the scan head array to guarantee that the sampling sites were independent. All deformations were performed freehand while captured data were visually inspected in real time on specifically designed research mode software created by T. Hall and Y. Zhu (University of Kansas Medical Center, Kansas City, KS) and implemented on the Siemens Sonoline Elegra system. ${ }^{12}$ During each deformation, the operators closely monitored the stability of the speckle pattern within each thrombus. If there was a subjective rapid fluctuation of the pattern while deforming, the deformation was repeated. We acquired approximately 240 frames of phase-sensitive data after an approximately 1$\mathrm{cm}$ surface displacement at each scanning plane. These data were stored on a personal computer for offline processing.

In all data sets, frame-to-frame motion was estimated by a short time correlation method that used two-dimensional, phase-sensitive speckle tracking as described previously. ${ }^{13-16}$ This particular technique combines the ability of correlation-based algorithms to track large displacements with the precision of phase-sensitive techniques. The in-plane frame-to-frame displacement vector components were accurately measured from the position of the maximal correlation coefficient, where a $1.3-\mathrm{mm}$ (lateral) by $0.55-\mathrm{mm}$ (axial) correlation kernel was used. The axial displacement estimate was further refined by determining the zero crossing position of the phase of the analytic signal correlation, and displacement error was reduced with the use of a weighted correlation sum and by filtering spatially adjacent correlation functions before displacement estimation. ${ }^{15}$ Finally, errors due to speckle decorrelation were reduced by accumulation of incremental frame-to-frame displacements to produce final displacement and strain images 
with an increased signal-to-noise ratio. ${ }^{16}$ The normal axial strains were computed from accumulated axial displacements with the use of a simple one-dimensional difference filter along the axial direction for correlation windows separated by $0.8 \mathrm{~mm}$.

We display the data as two-dimensional strain images where the strain dynamic range is presented in color. The strain values are negative because the deformation squeezes the objects. The more negative the strain (i.e., the higher the magnitude), the softer the object. Because we did not know the applied force, all of the strain values were measured relative to other objects in the images. In addition, at certain positions, we generated one-dimensional strain profiles that ran perpendicular to the sonographic scan head through the clots. These profiles more precisely represent the magnitudes of the strain fluctuations within each thrombus. The profiles are analogous to A-mode sonographic scans, in which each is a one-dimensional representation of a sonographic property as a function of position. In an A-mode image, gray scale backscatter along a line is displayed as a function of depth. In a strain profile, relative strain is plotted as a function of position along a particular line.

\section{Results}

Typical images from the studies of the 2 patients are shown (Figs. 1 and 2). We also show strain profiles through the respective thrombi (Figs. 3 and 4). Note that compared with the subacute thrombus in patient 2 , the strain profile through the chronic DVT in patient 1 is inverted; i.e., the strain magnitude in the chronic clot is smaller than that in the vessel wall, signaling that the clot is harder than the vessel wall (Fig. 3). Also note that this clot is relatively homogeneous, at least compared with the subacute thrombus in patient 2 . The subacute thrombus is inhomogeneous and complex (Figs. 2 and 4).

\section{Discussion}

Assessing the age of DVTs is important for therapeutic reasons. Acute thrombi are primarily composed of platelets, fibrin, and white blood cells and are considered unstable. They are, therefore, treated with heparin, a highly potent, fast-acting injected anticoagulant. However, because of heparin's potency, there is a risk of

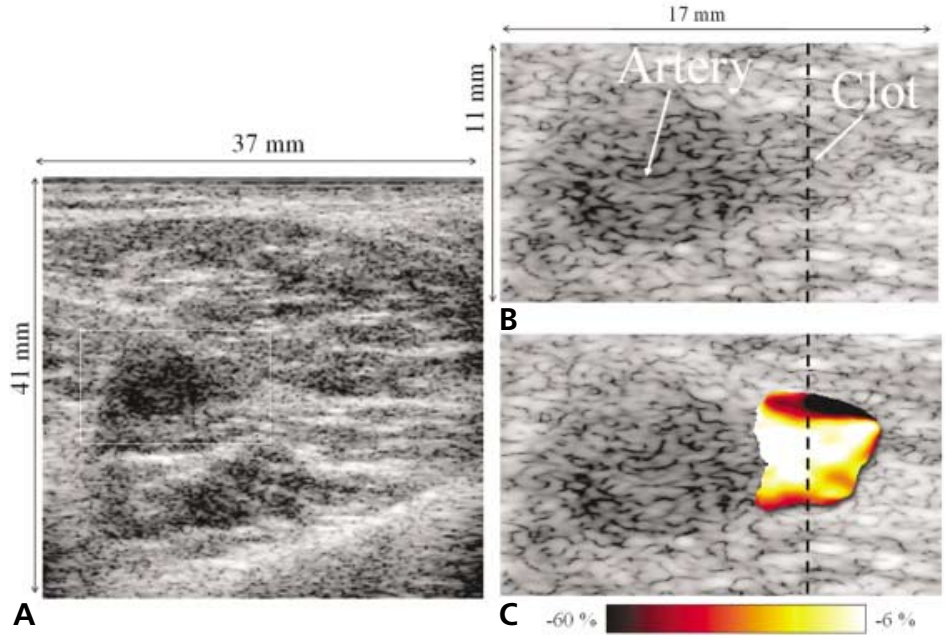

Figure 1. A, Transverse image of a lower leg in patient 1 with chronic DVT. The area of the popliteal artery and vein is marked with a white box. B, Magnification of the area surrounded by the white box in $\mathbf{A}$. The artery and vein are labeled and marked with arrows. The dotted line passing through the vein represents the path along which the strain profile is plotted in Figure 3. C, Two-dimensional strain image of the venous thrombus. The relative strains are displayed in color, with the dynamic range displayed as a color bar at the bottom. The more negative the strain, the softer the object. Clearly in this case, the center of the thrombus is generally harder than the edges. Again, the dotted line represents the path along which the strain profile in Figure 3 is calculated.

Figure 2. A, Gray scale image of a subacute saphenous vein clot in patient 2, with the 2 dotted lines corresponding to paths along which strain profiles were generated. Note that the clot is heterogeneous in the gray scale image, with a relatively hypoechoic center and a more echogenic margin. B, Two-dimensional color strain image of the clot shown in $\mathbf{A}$. The relative strains are displayed with the use of the color map as indicated in the color bar at the bottom. Again, the more negative the strain, the softer the structure. Note that this clot is much more heterogeneous than the chronic thrombus. The 2 dotted lines passing through the clot indicate paths along which the strain profiles were taken. The vertical grid between $\mathbf{A}$ and $\mathbf{B}$ is in millimeters.
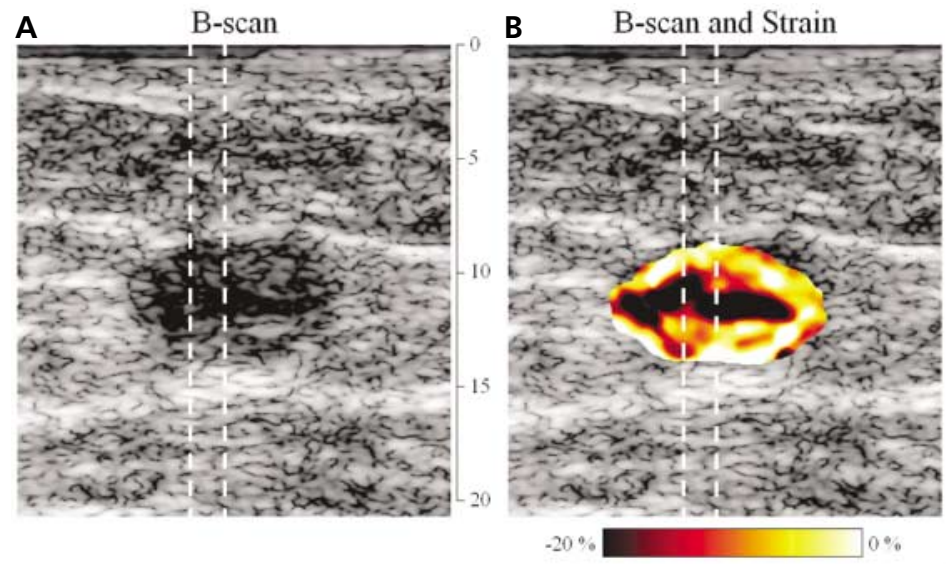


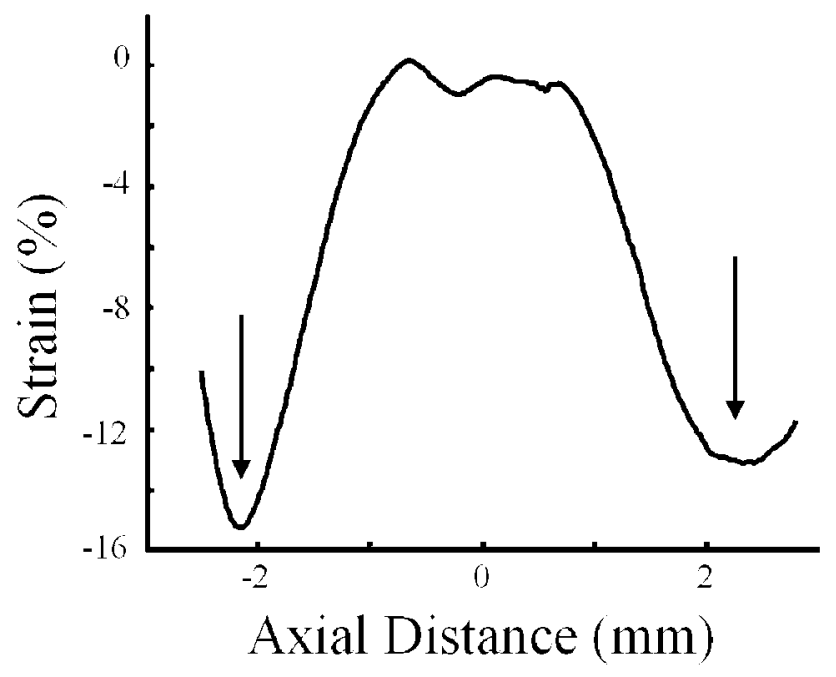

Figure 3. One-dimensional strain profile calculated along the path through the venous thrombus shown in Figure $1, B$ and $C$. The profile is centered the middle of the thrombus, and the vessel walls (arrows) are marked at about $\pm 2 \mathrm{~mm}$ from the center of the thrombus. Note that there is a strain inversion here; the strain magnitude in the clot is less than the strain magnitude in the vessel wall; that is, the clot is harder than the vessel walls. On the basis of this plot, the strain magnitude in the thrombus is 13 to 15 times smaller than that in the wall.

bleeding complications, some of which can even be fatal. As a clot matures, the fibrin is replaced with collagen, and the cells change from neutrophils to mononuclear cell types. ${ }^{6,7}$ These more mature clots are more stable. Ultimately chronic clots are, therefore, treated with warfarin sodium, which is safer than heparin and can be taken orally. Each stage in the maturation process increases the hardness of a thrombus, suggesting that hardness should be a good parameter for assessing clot maturity. This notion also is consistent with the classic physical diagnosis finding of a "rope" in the thigh in patients with chronic superficial thrombosis.

Given the above, a technique that assesses hardness (i.e., sonographic elasticity imaging) should be ideal for determining clot maturity. In addition, sonographic elasticity imaging blends perfectly with compression sonographic scanning, the present standard diagnostic technique for DVT, because both require that the operator push over the veins of the legs and watch the deformation.

We studied 2 patients with clots of different ages. Patient 1 had long-standing chronic DVT. This clot was homogeneous, and its profile appeared remarkably similar to that of an analogous 9-day-old inferior vena cava thrombus (chronic DVT) that we generated in a rat-based animal model of stasis-induced DVT (Fig. 5). ${ }^{11}$ Both of these clots had strain inversion, with the clot becoming harder than the vessel wall. In the region displayed in the strain profile, the strain magnitude in patient 1's thrombus was about 13 to 15 times smaller than that in the vessel wall.

The subacute clot in patient 2 was much more complex, with areas that were nearly as hard as

Figure 4. A, Strain profile taken along the left path. Notice that the strain is highly variable in this complex clot. If the wall of the vein is identified at the positions marked (arrows), the strain magnitude in the soft portion of the clot can be as much as 4 to 30 times higher than that in the wall. B, Strain profile taken along the right path shown in Figure 3. Again, the heterogeneity of the thrombus is shown, where arrows mark the vessel wall. The more superficial portion of the thrombus is relatively hard, but the softest part of the clot exhibits a strain magnitude on the order of 9 to 40 times higher than that in the vessel wall.

A

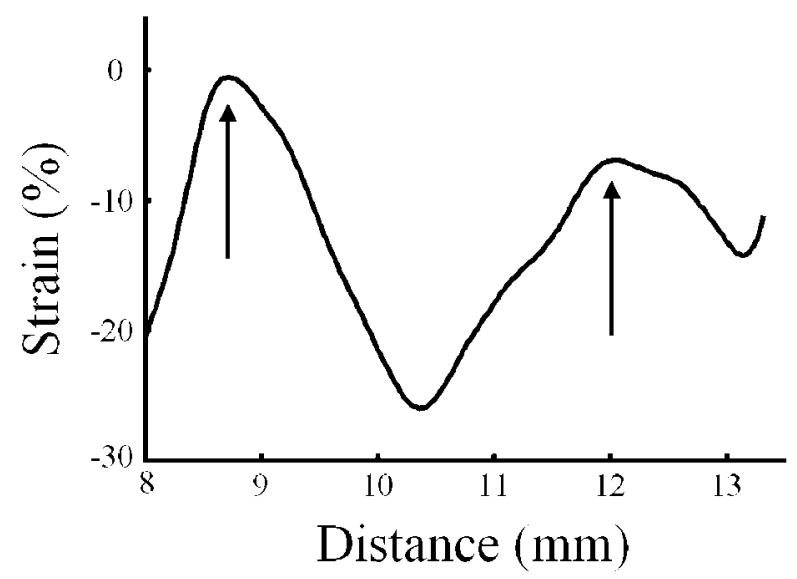

B

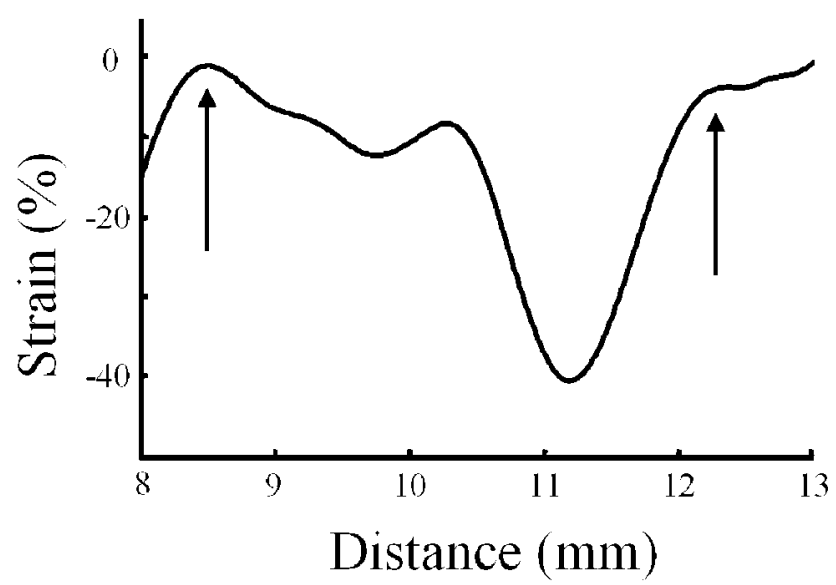


the vessel wall and other areas that were much softer. Similar strain profiles and complexity were also seen in softer (i.e., acute or subacute) clots in rats ${ }^{11}$ as, for example, presented in Figure 5 . The ability of elasticity imaging to detect complex hardness patterns in thrombi should be useful for assessing therapy as well as for monitoring thrombus changes during different therapeutic regimens.

If it is assumed that the vessel walls had the same hardness in both patients, the chronic clot was conservatively at least 2 times harder than the subacute clot. However, in a comparison of the softest part of the subacute clot and the chronic clot, it is possible that the chronic clot was many times harder than the subacute clot (Figs. 3 and 4).

An additional property of elasticity imaging is that the degree of deformation required to make a measurement is much smaller than what is typically used in compression sonography. ${ }^{9-11}$ Compression sonography generally requires complete collapse of either the femoral vein or an adjacent artery to diagnose DVT. ${ }^{1}$ This equates to nearly $100 \%$ strain in one of the vessels. Conversely, standard strain measurements on sonographic elasticity imaging can easily be done with the use of strains on the order of a few percent. ${ }^{15,16}$ We used larger strains to improve the signal-to-noise ratio, ${ }^{16}$ but such large strains are not required. Hence, sonographic elasticity imaging requires a much smaller push compared with standard compression sonography. This could be a big benefit in patients with swollen, painful legs. Hence, even if evaluation of clot age does not work, just the use of sonographic elasticity imaging to detect thrombi may be an improvement over the present compression sonographic technique.

The main limitation of this study was that it was very preliminary, consisting of only 2 patients. However, the results are consistent with earlier experimental work on an animal model of DVT and with what one would expect knowing the normal evolution of thrombi. , $^{8,11}$

Another limitation is that comparison between the 2 clots requires an internal standard with the same hardness in both images, because we do not know the force that was used to deform the tissue in each case. We have typically used the wall of the vein as this standard. ${ }^{11}$ On the basis of the profile in the chronic DVT case (Fig. 3), it is easy to see where the wall might be. However, in the case of the subacute clot, the walls are much more poorly defined. We know approximately where the walls are, but the ultimate uncertainty in defining the position of the wall only makes it possible to grossly compare the 2 thrombi. The venous wall does, however, change over time with DVTs. This is due to the well-known propensity for fibro-intimal reactions in veins to literally absorb chronic thrombi into the venous wall. ${ }^{17}$ In addition, both the venous wall and the thrombus undergo typical acute to chronic inflammatory responses. ${ }^{6,18}$ Consequently, both the thrombus and wall are hardening as DVT develops and matures within the vein. Hence, we could expect that both veins and their respective thrombi in our study would harden with time, yet because the chronic DVT was harder than the wall, and the subacute DVT was either almost the same hardness as or softer than the wall, the hardness difference between the 2 thrombi was actually greater than what one would predict assuming that the venous walls were identical in hardness. In other words, our hardness difference estimate is actually conservative. Of course, one could always use another reference such as the arterial wall. This would not have worked in our case, because the saphenous vein does not

Figure 5. Strain profiles drawn through the centers of 2- and 9-day-old inferior vena cava thrombi in 2 different rats. In this accelerated model of DVT, these clots would correspond to acute or subacute and chronic DVT in humans. Notice the strain inversion relative to the vessel walls (dotted arrows) in the 9-day-old clot (chronic DVT). This corresponds closely to the shape of the chronic DVT in Figure 3. The solid arrows mark the likely positions of the vessel walls in the 2-day-old thrombus, showing that the 2-day-old clot is much softer than the vessel wall. Also note the complexity of the 2-day-old clot, represented by the nearly 10\% strain difference across its center (dashed arrows). Adapted with permission from Ultrasound in Medicine and Biology. ${ }^{11}$

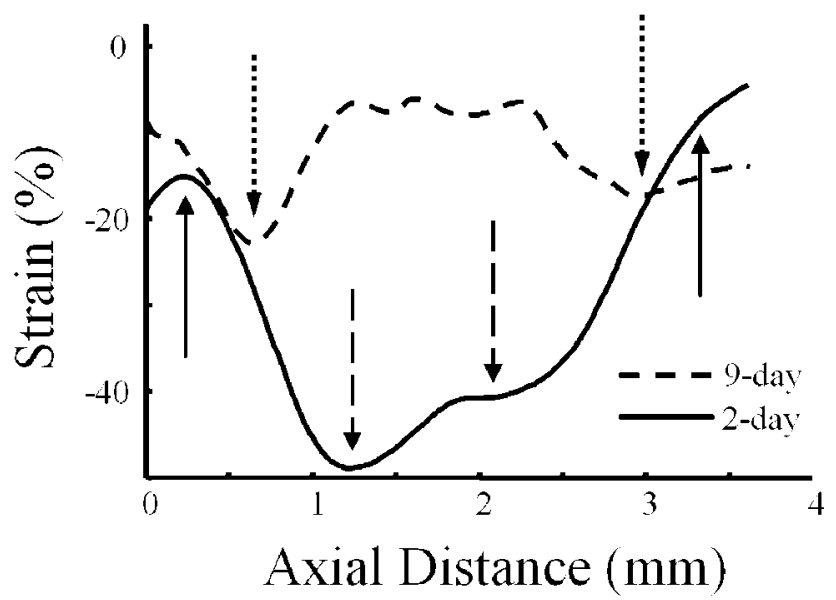


have an associated artery. Comparing the hardness with that of the venous wall should be valid, however, because the inflammatory response involving thrombi and venous walls appears to be independent of whether a vein is in the deep or superficial system. ${ }^{18}$ This suggests that thrombi in both systems have similar rates of hardening.

The solution to these problems is to either reconstruct Young's modulus of the thrombi using a direct ${ }^{19}$ or model-based approach ${ }^{20}$ or to actually acquire the force used during a deformation. ${ }^{21}$ We are planning on making such measurements in the future. Even without knowledge of precisely where the wall is, however, each clot's strain image does accurately represent that particular clot's complexity. Hence, elasticity imaging has the potential for revealing new information about the evolution of thrombi with and without therapy.

Further investigation is required. However, given these preliminary results, we think that sonographic elasticity imaging has potential for improving the assessment and treatment of DVT.

\section{References}

1. Lewis BD. The peripheral veins. In: Rumack CM, Wilson SR, Charboneau JW (eds). Diagnostic Ultrasound. 2nd ed. St Louis, MO: CV Mosby Co; 1998:946-954.

2. Douglas MG, Sumner DS. Duplex scanning for deep vein thrombosis: has it replaced both phlebography and noninvasive testing? Semin Vasc Surg 1996; 9:3-12.

3. Prandoni P, Cogo A, Bernardi E, et al. A simple ultrasound approach for detection of recurrent proximalvein thrombosis. Circulation 1993; 88:1730-1735.

4. Fraser JD, Anderson DR. Deep venous thrombosis: recent advances and optimal investigation with US. Radiology 1999; 211:9-24.

5. Wakefield TW. Treatment options for venous thrombosis. J Vasc Surg 2000; 31:613-620.

6. Wakefield TW, Linn MJ, Henke PK, et al. Neovascularization during venous thrombosis organization: a preliminary study. J Vasc Surg 1999; 30:885-892.

7. Henke PK, Wakefield TW, Kadell AM, et al. Interleukin8 administration enhances venous thrombosis resolution in a rat model. J Surg Res 2001; 99:84-91.

8. Browse NL, Burnand KG, Irvine AT, Wilson NM. Deep vein thrombosis: pathology. In: Browse NL, Burnand
KG, Irvine AT, Wilson NM (eds). Disease of the Veins. 2nd ed. London, England: Arnold; 1999:249-289.

9. Ophir J, Cespedes I, Garra B, Ponnekanti H, Maklad N. Elastography: ultrasonic imaging of tissue strain and elastic modulus in vivo. Eur J Ultrasound 1996; 3:49-70.

10. Gao L, Parker KJ, Lerner RM, Levinson SF. Imaging of the elastic properties of tissue: a review. Ultrasound Med Biol 1996; 22:959-977.

11. Emelianov SY, Chen X, O'Donnell M, et al. Triplex ultrasound: elasticity imaging to age deep venous thrombosis. Ultrasound Med Biol 2002; 28:757-767.

12. Hall TJ, Zhu Y, Spalding CS. In vivo real-time freehand palpation imaging. Ultrasound Med Biol. In press.

13. O’Donnell M, Emelianov SY, Skovoroda AR, Lubinski MA, Weitzel WF, Wiggins RC. Quantitative elasticity imaging. Proc IEEE Ultrason Symp 1993; 2:893-903.

14. O'Donnell M, Skovoroda AR, Shapo BM, Emelianov SY. Internal displacement and strain imaging using ultrasonic speckle tracking. IEEE Trans Ultrason Ferroelectr Freq Control 1994; 41:314-325.

15. Lubinski MA, Emelianov SY, O’Donnell M. Speckle tracking methods for ultrasonic elasticity imaging using short time correlation. IEEE Trans Ultrason Ferroelectr Freq Control 1999; 46:82-96.

16. Lubinski MA, Emelianov SY, O'Donnell M. Adaptive strain estimation using retrospective processing. IEEE Trans Ultrason Ferroelectr Freq Control 1999; 46:97-107.

17. Murphy TP, Cronan JJ. Evolution of deep venous thrombosis: a prospective evaluation with US. Radiology 1990; 177:543-548.

18. Wakefield TW, Strieter RM, Prince MR, Downing $\sqcup$, Greenfield $\amalg$. Pathogenesis of venous thrombosis: a new insight. Cardiovasc Surg 1997; 5:6-15.

19. Skovoroda AR, Emelianov SY, O'Donnell M. Reconstruction of tissue elasticity based on ultrasound displacements and strain images. IEEE Trans Ultrason Ferroelectr Freq Control 1995; 42:747-765.

20. Aglyamov SR, Skovoroda AR, Rubin JM, O'Donnell $M$, Emelianov SY. Young's modulus reconstruction in DVT elasticity imaging. Ultrason Imaging 2002; 20(suppl):174-175.

21. Sarvazyan AP. Mechanical imaging: a new technology for medical diagnostics. Int J Med Inf 1998; 49: 195-216. 